\title{
An improved vision after treatment of endophthalmitis following keratoprosthesis - a report of two cases
}

\author{
Farheen Fatima', Amna Anwar', Farah $\mathrm{Naz}^{3}$, Azam Ali' \\ 'Section of Ophthalmology, Department of Surgery, Aga Khan University Hospital, Stadium Road, Karachi, Pakistan \\ ${ }^{2}$ Jinnah Medical College Hospital, Karachi, Pakistan \\ ${ }^{3}$ Dha Suffa University, DHA, Karachi, Pakistan
}

\begin{abstract}
Endophthalmitis following keratoprosthesis could lead to permanent loss of vision and the eye. It needs systematic management and close follow-ups as the outcome is unpredictable.

We present two cases of endophthalmitis following type I Boston keratoprosthesis (KPro). Both of our patients had lost one eye while the other eye developed endophthalmitis after they underwent prosthokeratoplasty. They were aggressively treated with topical and oral antibiotics with close follow-ups. Vitreous cultures were obtained, and intravitreal antibiotics were given. The endophthalmitis resolved with a dramatically improved vision.
\end{abstract}

KEY WORDS: endophthalmitis; keratoprosthesis (KPro)

Ophthalmol J 2021; Vol. 6, 61-64

\section{INTRODUCTION}

Although conventional penetrating keratoplasty (PKP) is a well-known treatment for several corneal diseases, some conditions are not amenable to PKP. The Boston keratoprosthesis (KPro) has been used as a treatment option in patients with multiple graft failure, Stevens-Johnson syndrome (SJS) [1], ocular cicatricial pemphigoid (OCP), several autoimmune diseases [2], and ocular burns (acid and alkali) [3, 4]. The three most commonly reported postoperative complications with keratoprosthesis are: retroprosthetic membrane (RPM), elevated intraocular pressure/glaucoma, and infectious endophthalmitis (IE) [5-8].

This article presents two cases of patients who developed endophthalmitis in the eye with vision following KPro. Patients were presented to us with very low vision. After the treatment, their vision was much improved.

\section{CASE PRESENTATION \\ Patient no. 1}

A 52-year-old male was referred to a retina specialist following type I Boston keratoprosthesis, which was conducted two weeks earlier by a local corneal specialist. The patient complained of redness and discharge from the right eye. The eye underwent prosthokeratoplasty for multiple graft failures. Past history showed that the patient suffered from corneal decompensation in both eyes. The multiple PKPs were performed, but the grafts were

\section{CORRESPONDING AUTHOR:}

Farheen Fatima, Section of Ophthalmology, Department of Surgery, Aga Khan University Hospital, Stadium Road, Karachi, Pakistan; e-mail: dr.farheenfatima5@gmail.com 

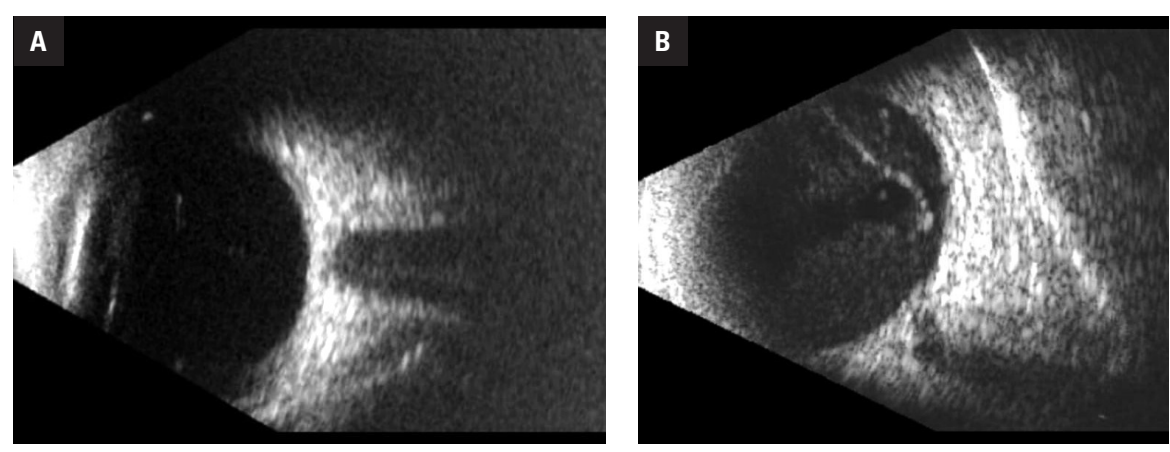

FIGURE 1. Patient no. 1. A. B-scan of the right eye showing significant vitriti; B. B-scan of the right eye of the same patient showing resolution of vitritis after the treatment

rejected, and the patient had lost his vision completely in the left eye. The redness in the right eye followed by a copious and sticky discharge appeared a week before the visit.

On examination, the visual acuity test results showed counting fingers in the right eye and no perception of light in the left eye. Digital palpation revealed normal globe contour and intraocular pressure in the right eye. Anterior segment examination showed that the right eye had Boston KPro in place with severe ciliary congestion and mucopurulent discharge. The B-scan of the right eye showed significant vitritis and no retinal detachment (Fig. 1A).

The treatment included: eye drops moxifloxacin $0.5 \%$, tobramycin $0.3 \%$ + dexamethasone $0.1 \%$, amikacin, and vancomycin hourly, along with oral moxifloxacin $400 \mathrm{mg}$ once a day. After two days, the patient's vision further deteriorated (on visual acuity test scale to hand movements), so he was treated with intravitreal moxifloxacin, ceftazidime, amphotericin B, and dexamethasone. A vitreous tap was performed, and the undiluted vitreous sample was sent in a sterile syringe for Gram staining and culture test for both bacteria and fungi. Treatment with antibiotics was continued. Vitreous Gram staining, microbial culture, and sensitivity reports were negative for any bacterial and fungal growths. The patient was regularly followed up. The right eye examination showed that his vision gradually improved to $20 / 60$, ciliary congestion subsided, and mucopurulent discharge was completely resolved. Repeated B-scan showed resolution of vitritis (Fig. 1B). Eye drops were gradually tapered off, and his vision remained stable.

\section{Patient no. 2}

A 75-year-old female was referred to a retina specialist following type I Boston keratoprosthe- sis (KPro). The procedure was performed a week earlier by a local cornea specialist. The patient had been experiencing excruciating pain. The redness and discharge have been present since after the procedure. Past history showed that the patient suffered from Fuch's endothelial dystrophy in both eyes and underwent cataract surgeries 15 years earlier. Since after the cataract surgeries, the patient had been experiencing a dropped vision in both eyes, for which she had multiple PKP performed, after which she lost her vision entirely in her right eye. The last grafting procedure was conducted two years ago in the left eye. Still, the patient's vision dropped, so, with the hope of having improved vision, she underwent prosthokeratoplasty, which took place a week before referral to our clinic.

On examination, the visual acuity test showed no light perception in the right eye and light perception in the left eye. Digital palpation revealed normal globe contour and intraocular pressures in the left eye. Anterior segment examination showed that the left eye had Boston KPro in place with severe ciliary congestion and mucopurulent discharge. $\mathrm{B}$-scan of the left eye showed significant vitritis and no retinal detachment (Fig. 2A).

Initially, the patient was treated with eye drops amikacin, vancomycin, and prednisolone acetate hourly, along with oral moxifloxacin $400 \mathrm{mg} O D$ and amoxycillin and clavulanic acid (Augmentin) $1 \mathrm{~g} B I D$. A vitreous tap was performed, and an undiluted vitreous sample was sent in a sterile syringe for Gram staining, culture test, and sensitivity test for bacteria and fungi. Intravitreal antibiotics: amikacin, ceftazidime, moxifloxacin, and dexamethasone were applied to the left eye. Vitreous sample reports were negative for bacterial and fungal growths. On subsequent visits, patient's vision improved (on visual acuity test scale to counting 

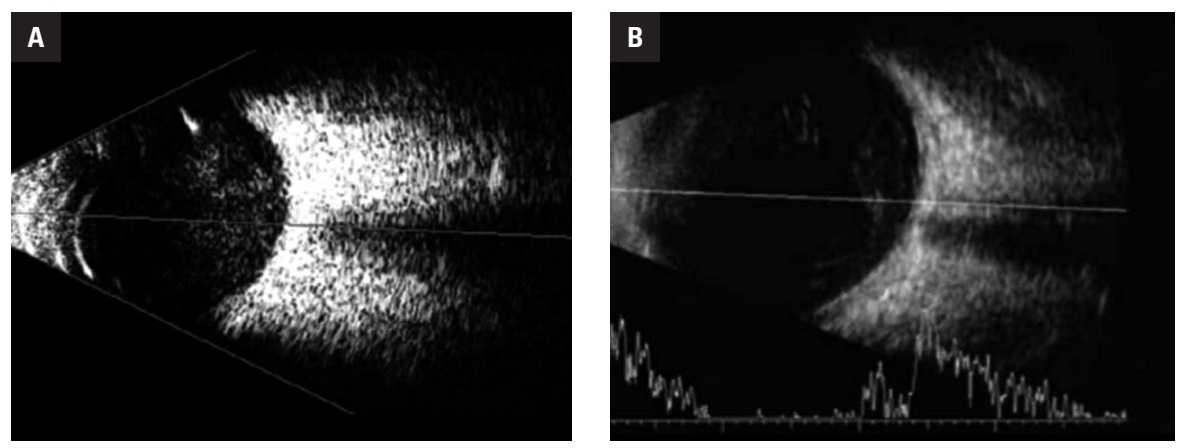

FIGURE 2. Patient no. 2. A. B-scan of the left eye showing significant vitritis; B. B-scan of the left eye of the same patient showing resolving vitritis after the treatment

finger), Kpro was in place, the inflammation and mucopurulent discharge gradually subsided. Posterior chamber intraocular lens (PC IOL) was in place with posterior capsular opacification (PCO). B-Scan showed resolving vitritis (Fig. 2B). Eye drops were gradually tapered off.

Four weeks later, Nd:Yag capsulotomy was performed for PCO. Following laser treatment, the patient's vision improved to $20 / 400$, which remained stable.

\section{DISCUSSION}

Endophthalmitis following keratoprosthesis accounts for the overall incidence of $2.7 \%$ of patients per year according to the most comprehensive case series [9]. This serves to be one of the highest endophthalmitis rates.

Endophthalmitis is one of the devastating complications of keratoprosthesis and needs immediate care by means of topical, systemic, and intravitreal antibiotics. Vitreous cultures also need to be performed to isolate the growth of bacteria and fungi, which helps select appropriate antibiotics and aid in-line treatment.

Once a case of endophthalmitis is diagnosed, it needs systematic management together with a closer follow-up. The management remains a challenge due to the lack of evidence and the exceptionality of the examination and therapeutic approach. Georgalas et al. have previously reported three cases of endophthalmitis following keratoprosthesis, which were treated with 25 gauge pars plana vitrectomy. The overall outcome of this treatment was good [10].

Both of our patients had a vision in one eye only. Both underwent prosthokeratoplasty with type I Boston KPro for multiple graft rejections with the hope of gaining improved vision, but unfortunately, they developed endophthalmitis. Patients were aggressively treated with broad-spectrum antibiotics.

The treatment not only saved their vision but also improved the vision to the extent that they also gained a better quality of life.

\section{Statement of ethics}

The patient provided written, informed consent for submission of the case report and any accompanying images. This case report was approved by the Ethics Committee of Aga University Hospital, Karachi, Pakistan.

\section{Disclosure statement}

The authors declare that they have no conflicts of interest.

\section{REFERENCES}

1. Pineda R. The Boston keratoprosthesis in Stevens-Johnson syndrome. Evidence-Based Ophthalmol. 2008; 9(3): 166-167, doi: 10.1097/ ieb.0b013e31817d89a9.

2. Ciralsky J, Papaliodis GN, Foster S, et al. Keratoprosthesis in autoimmune disease. Ocul Immunol Inflamm. 2010; 18(4): 275-280, indexed in Pubmed: 20662659.

3. Tuft SJ, Shortt AJ. Surgical rehabilitation following severe ocular burns. Eye (Lond). 2009; 23(10): 1966-1971, doi: 10.1038/eye.2008.414, indexed in Pubmed: 19169226.

4. Harissi-Dagher M, Dohlman CH. The Boston Keratoprosthesis in severe ocular trauma. Can J Ophthalmol. 2008; 43(2): 165-169, doi: 10.3129/ i08-009, indexed in Pubmed: 18347618.

5. Chew HF, Ayres BD, Hammersmith KM, et al. Boston keratoprosthesis outcomes and complications. Cornea. 2009; 28(9): 989-996, doi: 10.1097/IC0.0b013e3181a186dc, indexed in Pubmed: 19724214.

6. Bradley J, Hernandez E, Schwab I, et al. Boston Type 1 Keratoprosthesis: The University of California Davis Experience. Cornea. 2009; 28(3): 321-327, doi: 10.1097/ico.0b013e31818b8bfa, indexed in Pubmed: 19387235.

7. Aldave A, Kamal K, Vo R, et al. The Boston Type I Keratoprosthesis. Ophthalmology. 2009; 116(4): 640-651, doi: 10.1016/j. ophtha.2008.12.058, indexed in Pubmed: 19243830.

8. Akpek EK, Harissi-Dagher M, Petrarca R, et al. Outcomes of Boston keratoprosthesis in aniridia: a retrospective multicenter study. Am J Ophthalmol. 2007; 144(2): 227-231, doi: 10.1016/j.ajo.2007.04.036, indexed in Pubmed: 17543875. 
9. Durand ML, Dohlman CH. Successful prevention of bacterial endophthalmitis in eyes with the Boston keratoprosthesis. Cornea. 2009; 28(8): 896-901, doi: 10.1097/IC0.0b013e3181983982, indexed in Pubmed: 19654525.
10. Georgalas I, Kanelopoulos AJ, Petrou P, etal. Presumed endophthalmitis following Boston keratoprosthesis treated with 25 gauge vitrectomy: a report of three cases. Graefes Arch Clin Exp Ophthalmol. 2010; 248(3): 447-450, doi: 10.1007/s00417-009-1230-3, indexed in Pubmed: 20012644. 